Journal of Applied Fluid Mechanics, Vol. 7, No. 2, pp. 329-344, 2014.

Available online at www.jafmonline.net, ISSN 1735-3572, EISSN 1735-3645.

DOI: $10.36884 / \mathrm{jafm} .7 .02 .20279$

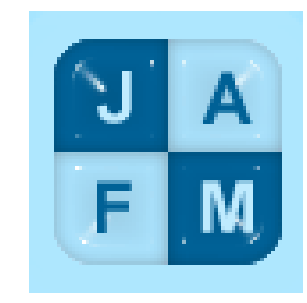

\title{
A Novel Aerodynamic Design Method for Centrifugal Compressor Impeller
}

\author{
M. Nili-Ahmadabadi ${ }^{1}$, M. Durali ${ }^{2}$ and A. Hajilouy ${ }^{2}$ \\ ${ }^{I}$ Department of Mechanical Engineering, Isfahan University of Technology, Isfahan, Iran \\ ${ }^{2}$ School of Mechanical Engineering, Sharif University of Technology, Tehran, Iran \\ †Corresponding Author Email: m.nili@cc.iut.ac.ir
}

(Received April 3, 2013; accepted May 9, 2013)

\begin{abstract}
This paper describes a new quasi-3D design method for centrifugal compressor impeller. The method links up a novel inverse design algorithm, called Ball-Spine Algorithm (BSA), and a quasi-3D analysis. Euler equation is solved on the impeller meridional plane. The unknown boundaries (hub and shroud) of numerical domain are iteratively modified by BSA until a target pressure distribution in flow passage is reached. To validate the quasi$3 \mathrm{D}$ analysis code, existing compressor impeller is investigated experimentally. Comparison between the quasi-3D analysis and the experimental results shows good agreement. Also, a full 3D Navier-Stokes code is used to analyze the existing and designed compressor numerically. The results show that the momentum decrease near the shroud wall in the existing compressor is removed by hub-shroud modifications resulting an improvement in performance by 0.6 percent.
\end{abstract}

Keywords: Centrifugal compressor, Inverse design, Quasi-3D, CFD, Ball spine Algorithm (BSA).

\section{NOMENCLATURE}

$\begin{array}{ll}\text { a } & \text { linear acceleration }\left(\mathrm{m}^{-\mathrm{s}^{-2}}\right) \\ \mathrm{A} & \text { local area of duct wall }\left(\mathrm{m}^{2}\right) \\ \mathrm{BSA} & \text { ball spine algorithm } \\ \mathrm{CPD} & \text { current pressure distribution on the wall } \\ \mathrm{CP}_{\mathrm{r}} \mathrm{D} & \text { current reduced pressure distribution } \\ \mathrm{F} & \text { force vector }(\mathrm{N}) \\ \mathrm{FSA} & \text { flexible string algorithm } \\ \mathrm{m} & \text { ball mass }(\mathrm{kg}) \\ \mathrm{n} & \text { number of virtual balls, normal to wall } \\ \mathrm{P} & \text { static pressure }(\mathrm{Pa}) \\ \mathrm{P}_{0} & \text { stagnation pressure }(\mathrm{Pa}) \\ \mathrm{P}_{0 \mathrm{r}} & \text { relative stagnation pressure }(\mathrm{Pa}) \\ \mathrm{P}_{\mathrm{r}} & \text { reduced pressure }(\mathrm{Pa}) \\ \mathrm{r} & \text { radial coordinate, radius }(\mathrm{m}) \\ \mathrm{SSD} & \text { surface shape design }\end{array}$

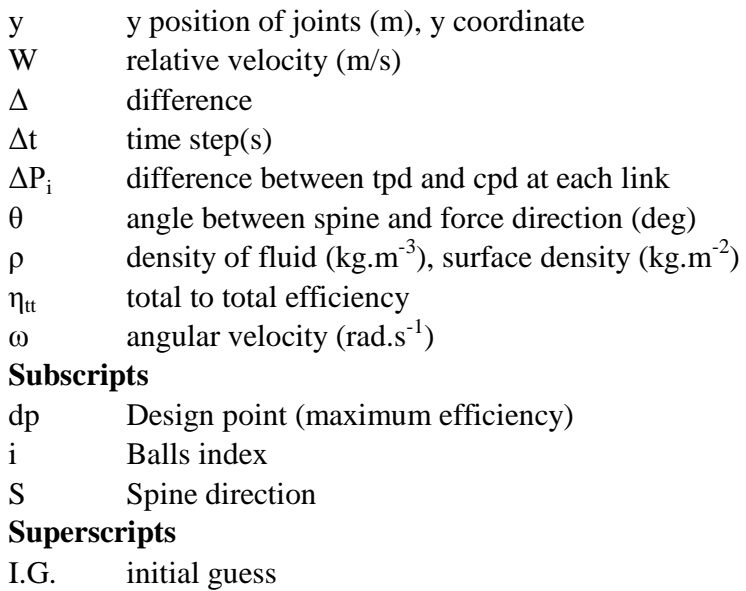




$\begin{array}{ll}\mathrm{t} & \text { tangent to wall } \\ \mathrm{TPD} & \text { target pressure distribution } \\ \mathrm{TP}_{\mathrm{r}} \mathrm{D} & \text { target reduced pressure distribution } \\ \mathrm{x} & \mathrm{x} \text { position of joints }(\mathrm{m}), \mathrm{x} \text { coordinate }\end{array}$

\section{INTRODUCTION}

Design of gas turbine components such as intakes, manifolds, duct reducers, and compressor and turbine blades involves shape determination of solid elements while fluid flow or heat transfer rate is optimal in some sense. The technological limitations and computational costs are challenging obstacles in such problems.

One of the known optimal shape design methods is Surface Shape Design (SSD). SSD in fluid flow problems involves finding a shape associated with a prescribed distribution of surface pressure or velocity. It should be mentioned that the solution of a SSD problem is not necessarily an optimum solution in mathematical sense. It just means that the solution satisfies a Target Pressure Distribution (TPD) which resembles a nearly optimum performance (Ghadak 2005).

There are basically two different algorithms for solving SSD problems namely decoupled (iterative) and coupled (direct or non-iterative). In the coupled solution approach, an alternative formulation of the problem is used in which the surface coordinates appear (explicitly or implicitly) as dependent variables. In other words, the coupled methods tend to find the boundary and flow field unknowns simultaneously in a (theoretically) single-pass or oneshot approach (Ghadak 2005).

The iterative shape design approach relies on repeated shape modifications such that each iteration consists of flow solution followed by a geometrical updating scheme. In other words, a series of sequential problems are solved in which the surface shape is changed between iterations till desired TPD is achieved (Ghadak 2005).

Iterative methods, such as optimization techniques, are widely used in solving practical SSD problems. The traditional iterative methods used for SSD problems are often based on trial and error algorithms. This trial and error process is very timeconsuming and computationally expensive. Hence it relies on designer experience for reducing the costs. Optimization methods (Cheng et al. 2000 and Jameson 1994) are used to automate the geometry modification in each iteration cycle. In such methods, an objective function (e.g., the difference between a current surface pressure and a target surface pressure (Kim et al. 2000)), subject to flow constraints is minimized. Although the iterative methods are general and powerful, they are often time consuming and mathematically complex. $\begin{array}{ll}\text { out } & \text { impeller outlet } \\ \mathrm{t}+\Delta \mathrm{t} & \text { related to updated geometry } \\ \mathrm{t} & \text { related to current geometry }\end{array}$

Other iterative methods presented so far, use the physical algorithms instead of the mathematical algorithms to automate the geometry modification in each iteration cycle. These methods are relatively easier and quicker. One of these physical algorithms is governed by a transpiration model in which one can assume the passage wall is porous. This implies that mass is fictitiously injected through the wall in such a way that the new wall satisfies the slip boundary condition. Aiming the elimination of nonzero normal velocity at the boundaries, a geometry update must be adopted. This update is determined by applying either the transpiration model based on mass flux conservation (Dedoussis et al. 1993, Demeulenaere et al. 1998, De Vito et al. 2003, Leonard et al. 1997 and Henne 1980), or streamline model based on alignment with the streamlines (Volpe 1989).

An alternative algorithm is based on the residualcorrection approach. In this method,the key problem is to relate the calculated differences between the ual pressure distribution on the estimated geometry act to the required changes in (the residual)and the TPD the art in developing a , Obviously the geometry correction method-residualis to find an optimumstate determining the for)between the computational effort and the number of (required geometry correction This .iterations needed to obtain a converged solution geometry correction may be estimatedby means of a making use of relations, simple correction rule differences between geometry changes and pressure known from linearized flow theory(Ghadak 2005).

correction decoupled solution methods -The residual .box-try to utilize the analysis methods as a black Barger et al. (1974)streamline curvature presented a method in which they considered ossibility of the $p$ relating a local change insurfacecurvature to a change

,Since then in local velocitya largenumber of methods have been developed on the basis of that concept (Garabedian et al. 1982, Malone et al. 1986, 1985, Campbell et al. 1987, Bell et al. 1991, Malone et al. 1989, 1991, Takanashi et al. 1985, Hirose et al. 1987, Dulikravich et al. 1999.(

The role of quasi-3D flow analysis relative to the detailed aerodynamic design of impellers is described in Aungier (2000). The key feature that keeps the quasi-3D Euler codes firmly entrenched over available exact viscous CFD codes in almost all design systems is the computational speed. The procedure of quasi-3D flow analysis can generate a solution in a matter of seconds even on a personal computer of modest capability. Currently, this seems the only analysis method that a designer needs for an efficient, fast and detailed component design.

For centrifugal compressor impeller, comparison between quasi-3D flow analysis and experimental 
results shows good agreement, except near trailing and leading edges, where the experimental data shows higher velocities than those predicted by the analysis (Aungier 2000).

Vanco (1972) gives the results of quasi-3D flow analysis used to find the flow distribution in the meridional plane of a centrifugal compressor. This method solves the velocity gradient equations with the assumption of a hub-to-shroud mean stream surface. A set of fixed arbitrary straight lines from hub to shroud is used instead of normal lines called quasi-orthogonal lines. These lines are independent of streamline changes. The work then determines the velocities in the meridional plane of a back-swept impeller, a radial impeller, and a vaned diffuser, as well as approximate blade surface velocities.

Zangeneh et al. (1988) found the presence of a high loss region in the passage and pointed out that the high loss region is similar to the "jet/wake" phenomenon observed in centrifugal compressors. They indicated that the high loss region is generated by the secondary flows. This secondary flow moves the low momentum fluids under the action of reduced static pressure towards the corner of shroud-suction side near the trailing edge.

Recently, Nili et al. (2009, 2009 and 2008) have presented a novel inverse design method called Flexible String Algorithm (FSA). They developed this method for non-viscous compressible and viscous incompressible internal flow regime, but FSA was not capable of axisymmetric and 3D design cases.

In the current research, using the physical bases of FSA, a novel inverse design algorithm called BallSpine Algorithm (BSA), simpler to the FSA, is presented for duct design. In BSA compared to FSA, the unknown walls are composed of a set of virtual balls, instead of flexible string, that move freely along specified directions called spines. The difference between the target and current pressure distribution on walls, move the balls along the spines. Considering that the BSA is an iterative inverse design method, it should be incorporated into a flow analysis code. In this investigation, a quasi-3D code solving Euler equation on the meridional plane of a centrifugal compressor is combined with BSA. The computational tool is employed in decreasing the high loss region near the shroud wall by modifying the pressure distribution along the hub and shroud wall. The walls are changed during the process to satisfy the modified pressure distributions. Full 3-D NavierStokes solutions of the original and the modified compressors were then compared. To validate the solutions from fully 3D Navier-Stokes and quasi-3D codes, experimental investigation was performed at Sharif Gas Turbine Lab.

\section{FUNDAMENTALS OF METHOD}

A 2-D flexible duct whose walls are composed of a set of virtual balls, freely moving along a specified direction, is shown in Fig. 1. Passing fluid through the flexible duct causes a pressure distribution to be applied to the inner side of duct wall. If a target pressure distribution is applied to the outer side of each duct wall, the flexible wall deforms to reach to a shape satisfying the target pressure distribution on the inner side. In other words, the force due to the difference between the target and current pressure distribution at each point of the wall is applied to each virtual ball and force them to move. As the target shape is reached, this pressure difference logically vanishes. If a virtual ball moves in the applied force direction, the adjacent balls may collide together or move away from each other. This can disturb the wall modification procedure. To avoid this problem, the balls should freely move in specified directions called spine. In Fig. 1, the spines are the normal line connecting the balls with the same $\mathrm{x}$ position on the two walls. In other words, the horizontal length of the duct remains unchanged during the shape modification procedure. In duct inverse design problems, it is essential that a characteristic length remains fixed. The direction of spines depends on the fixed length. Therefore, for different ducts, the spines are differently defined. Another constraint on this wall modification process is keeping one fixed point on each wall. Typically, the starting point of each wall is fixed so that the duct inlet area remains fixed too.

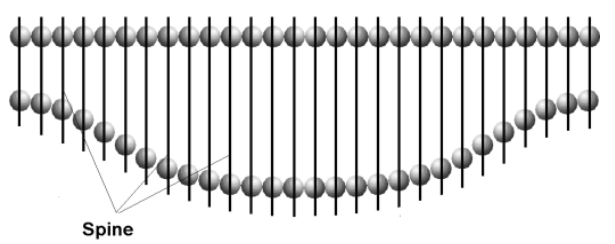

Fig. 1. Simulation of a 2-D duct with balls and spines.

\subsection{Governing Equations}

To derive kinematic relations of the flexible wall, a uniform mass distribution along the wall is assumed. Free body diagram of a virtual ball is shown in Fig. 2.

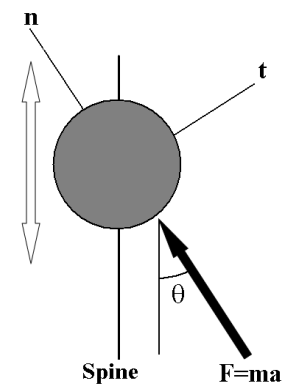

Fig. 2. Free body diagram of a ball.

The basic equations relations can be derived as follows:

$$
F_{S}=\Delta P \cdot A \cdot \cos \theta=m a_{S} \Rightarrow a_{S}=\frac{\Delta P \cdot A \cdot \cos \theta}{m}
$$




$$
\Delta y=\frac{1}{2} a_{S}(\Delta t)^{2}
$$

If $(\rho)$ is defined as wall surface density, the following relation can be written:

$$
\Delta y=\frac{1}{2} \frac{\Delta P \cdot A \cdot \cos \theta}{\rho A}(\Delta t)^{2}=\frac{(\Delta t)^{2}}{2 \rho} \Delta P \cos \theta
$$

$(\Delta t)$ is an interval for ball movement at each shape modification step. The parameter $\left(\Delta \mathrm{t}^{2} / \rho\right)$ is an adjusting parameter for the convergence rate of BSA method. The lower the value of $\left(\Delta \mathrm{t}^{2} / \rho\right)$, the slower the convergence rate will be. Indeed, if $\left(\Delta \mathrm{t}^{2} / \rho\right)$ exceeds a limit, the design algorithm will diverge. The new position of each ball for a horizontal duct as shown in Fig. 1 is obtained from the following relations:

$$
\begin{aligned}
& x_{i}^{(t+\Delta t)}=x_{i}^{(t)} \\
& y_{i}^{(t+\Delta t)}=y_{i}^{(t)}+\frac{(\Delta t)^{2}}{2 \rho} \Delta P_{i} \cos \theta_{i}
\end{aligned}
$$

In the meridional plane of a centrifugal compressor, the outlet radius instead of horizontal length should be fixed. In such geometry, the arbitrary straight lines from hub to shroud are the best choice for the spines. In Fig. 3, the schematic of ball displacement along the spine for the meridional plane is shown.

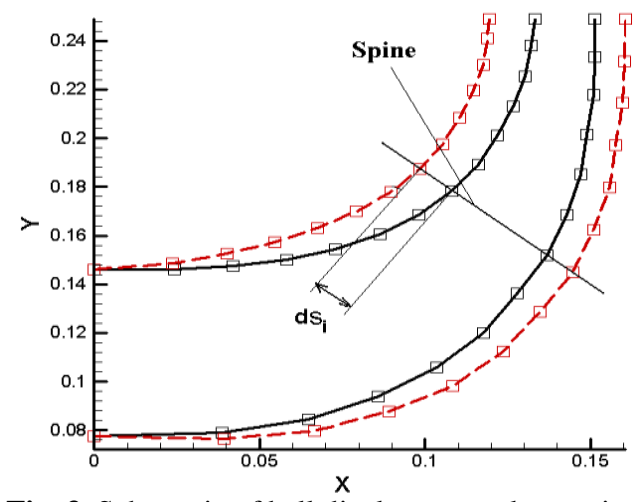

Fig. 3. Schematic of ball displacement along spine

Similar to other iterative inverse design methods, the flow field should be analyzed at each shape modification step. In this paper, the numerical techniques presented in (Zangeneh et al. 1988) are used for quasi-3D flow analysis. The spines used for inverse design are the same quasi-orthogonal lines used for quasi-3D analysis.

\subsection{Applying Pressure Difference to Each Virtual Ball}

As known, in inviscid flow, the stagnation pressure and relative stagnation pressure are constant along each stream line for stationary and rotating ducts, respectively.

$$
\begin{aligned}
& P_{0}=P+\frac{1}{2} \rho V^{2} \\
& P_{0 r}=P+\frac{1}{2} \rho W^{2}-\frac{1}{2} \rho r^{2} \omega^{2}
\end{aligned}
$$

Defining reduced pressure as follows:

$P_{r}=P-\frac{1}{2} \rho r^{2} \omega^{2}$

relative stagnation pressure can be rewritten as:

$P_{0 r}=P_{r}+\frac{1}{2} \rho W^{2}$

Analogy between Eq. (6) and (9) shows that the stagnation pressure, static pressure and velocity are replaced by the relative stagnation pressure, reduced pressure and relative velocity, respectively. In other words, reduced pressure is sensed from rotating duct wall similar to static pressure being sensed from stationary duct wall. Therefore, the growth of boundary layer thickness along the rotating duct wall depends on the reduced pressure gradient. Also, for inverse design of a rotating ducts such as centrifugal compressor meridional plane, the difference between the current and target reduced pressures is applied to each virtual ball on the wall. Finally, the displacement of each ball along its spine is obtained from the following equation:

$\Delta s_{i}=\frac{(\Delta t)^{2}}{2 \rho} \cos \theta_{i}\left[P_{r-\operatorname{target}}(i)-P_{r}(i)\right]$

Because the starting point of each wall should be fixed during the design procedure, the inlet reduced pressure is considered as inlet boundary condition for quasi-3D analysis code and remains fixed for the first virtual ball on the wall.

\section{BSA DESIGN PROCEDURE}

Figure 4 shows how BSA is typically combined with existing flow solution procedures. The computed pressure surfaces are normally obtained from partially converged numerical solutions of the flow equations. During the iterative design procedure, as the $\mathrm{CP}_{\mathrm{r}} \mathrm{D}$ approaches to the $\mathrm{TP}_{\mathrm{r}} \mathrm{D}$, the force applied to the flexible wall gradually vanishes. At final steps, the subsequent solutions of the flexible wall equations yield no changes in the duct surface coordinates.

\subsection{Quasi-3D Analysis}

To perform a quasi-3D analysis of a centrifugal compressor at its meridional plane, it is necessary to specify the input parameter. These quantities consist of mass flow rate, rotational speed, number of blades, specific-heat ratio, inlet total temperature and density, 
gas constant, hub-to-shroud profile, mean blade shape, and a normal thickness distribution of blades. Mass flow rate, rotational speed, inlet total temperature and density are obtained from experimental measurements at the compressor inlet at design point. The numerical values of the input parameters are specified in Table 1.

To determine the mean blade shape of the radial impeller, the angular coordinate of the mean blade shape is specified as a function of the axial distance, as shown in Fig. 5. Solution is started by an initial guess for streamlines. After the solution convergence, the streamlines are obtained as shown in Fig. 6. Figure 7 shows the reduced pressure distribution along the hub and shroud. Keen adverse gradient of the reduced pressure on the shroud causes the boundary layer thickness to grow.

In Fig. 8, the contour of static pressure is shown. As expected, the static pressure increases through the impeller. The contour of reduced pressure is shown in Fig. 9. Figure 10 shows the relative velocity contour. As can be seen in this figure, the maximum relative velocity occurs near the shroud at the inlet.

Table1 Centrifugal compressor parameters

\begin{tabular}{ll}
\hline Parameter(unit) & Value \\
\hline Operational condition & Design point \\
Number of impeller & 25 \\
Specific-heat ratio & 1.4 \\
Gas constant $(\mathrm{J} /(\mathrm{kg} . \mathrm{K}))$ & 287 \\
Inlet flow angle $\left({ }^{\circ}\right)$ & 0.0 \\
Inlet total temperature & 322 \\
Inlet total density & 1.088
\end{tabular}

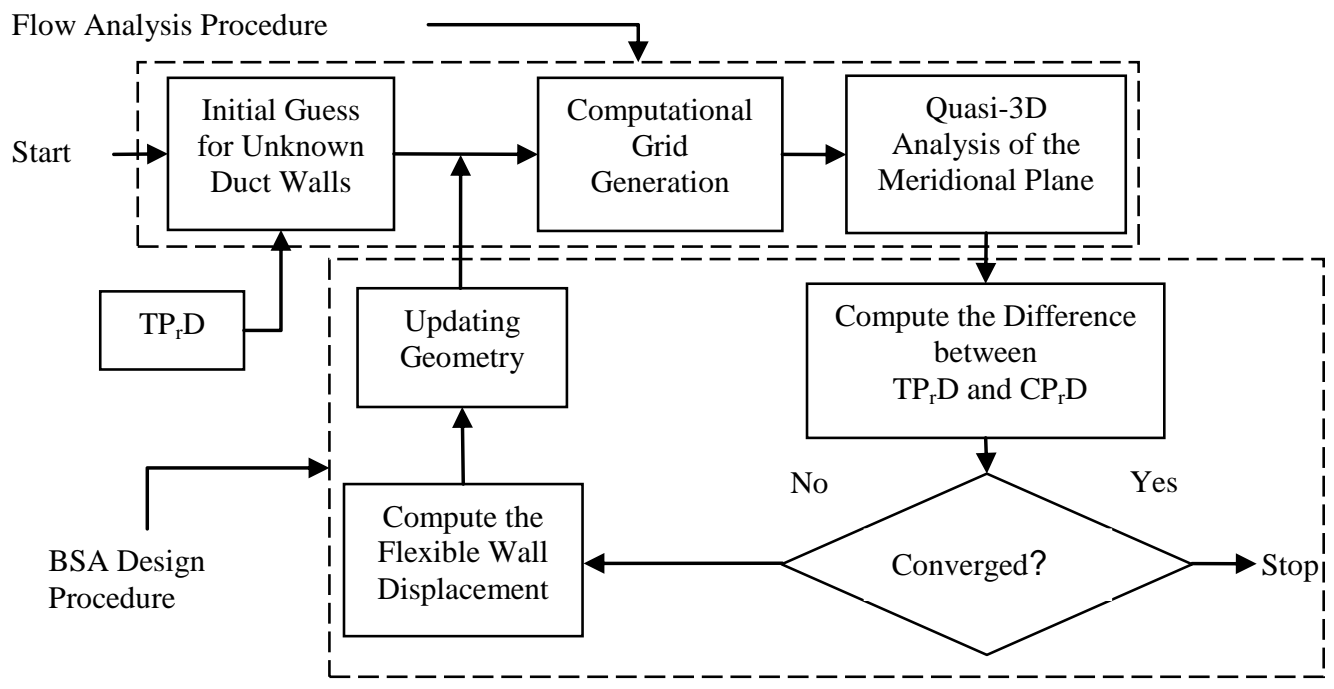

Fig. 4. Implementation of the inverse design algorithm

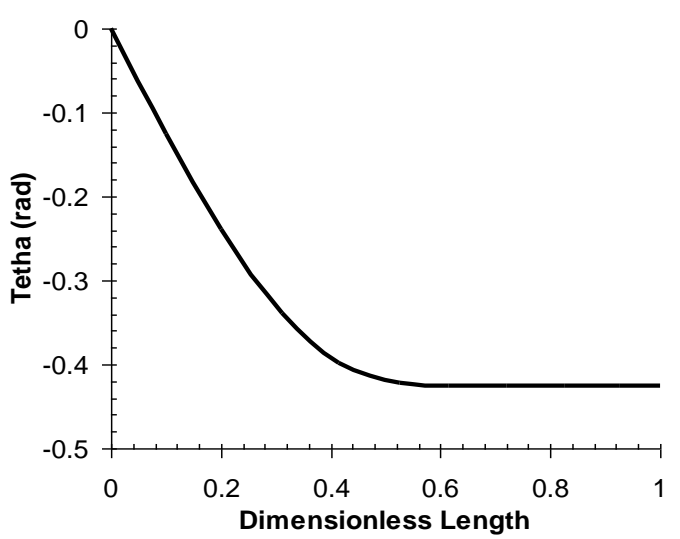

Fig. 5. Mean blade shape for radial impeller

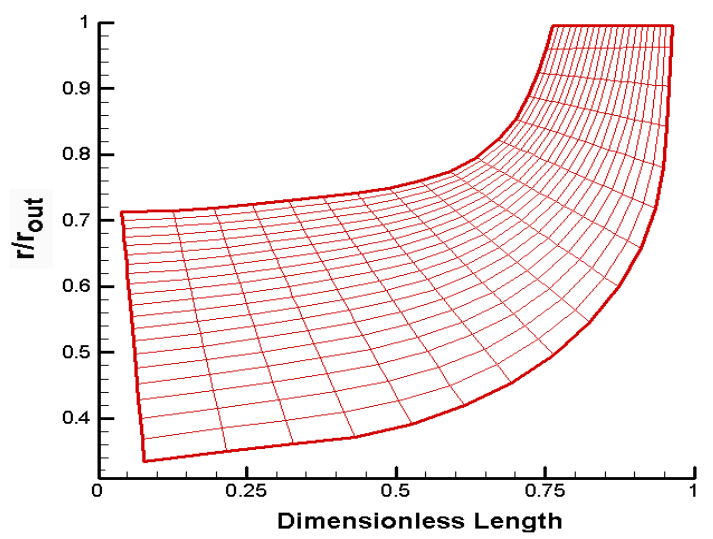

Fig. 6. Hub-to-shroud profile of radial impeller with its streamlines 


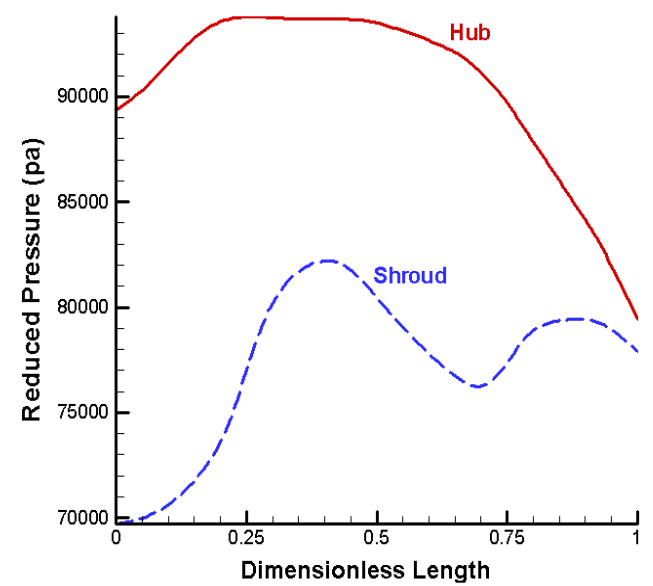

Fig. 7. Reduced pressure distribution along hub and shroud

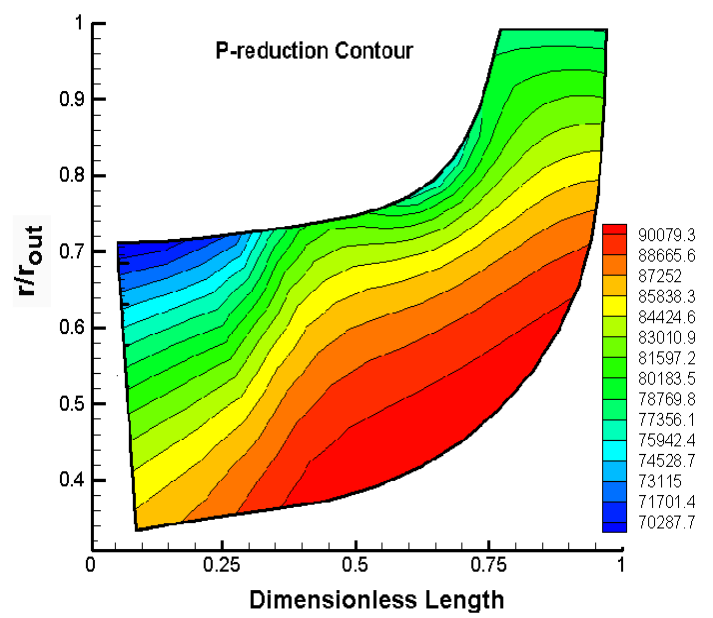

Fig. 9. Contour of reduced pressure on the meridional plane

\subsection{Inverse Design Validation Test Case}

For validation of the proposed method, the reduced pressure distributions along the hub and shroud in Fig. 7 are considered as our $\mathrm{TP}_{\mathrm{r}} \mathrm{D}$ for the SSD problems. The method should converge from an initial arbitrary shape to the shape shown in Fig. 6. Initiating from a meridional plane with larger area ratio, the design program algorithm is converged after 450 modification steps. Figure 11 shows the modification procedure from the initial guess to the target shape. The iterations are stopped after the design residual defined by the following equation reaches $10^{-3}$.

$$
\text { residual }=\frac{\sum_{i=1}^{N}\left[P_{i}-\left(P_{\text {Target }}\right)_{i}\right]}{\sum_{i=1}^{N}\left[\left(P_{\text {Target }}\right)\right]}
$$

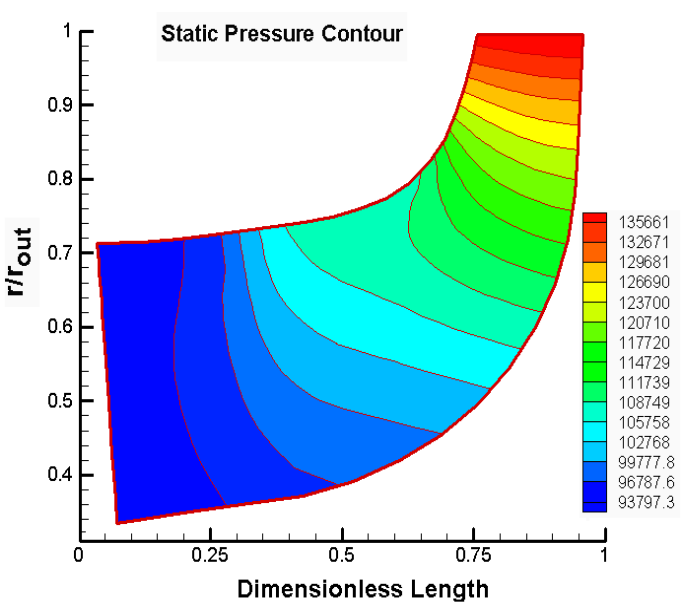

Fig. 8. Contour of static pressure on the meridional plane

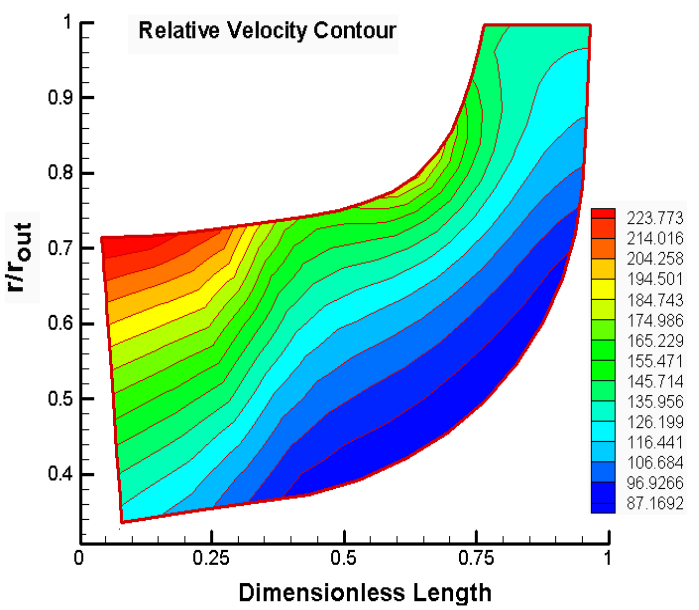

Fig. 10. Contour of relative velocity on the meridional plane

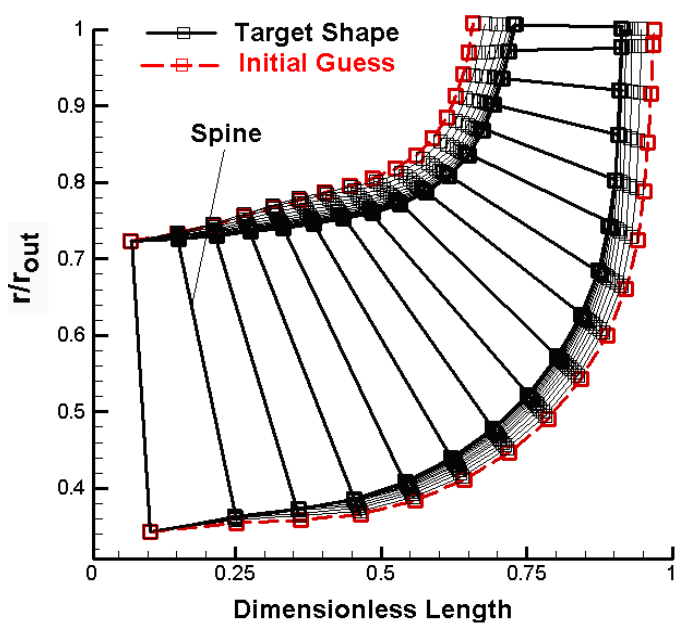

Fig. 11. Wall shape modification procedure 
After each geometry modification step, the quasi-3D analysis code is run until the residuals of the analysis code are reduced by 3 orders of magnitude. The residual of the analysis code is defined as the change in conserved flow variables $\left(\sum\left|\left(Q^{n+1}-Q^{n}\right) / Q^{\text {I.G. }}\right|\right)$ and is normalized by the residual of the first iteration. These convergence criteria for the design algorithm and analysis code are enough to confirm the required convergence so that the difference between calculated and target shapes cannot be recognized. Additional reduction of the residuals will just increase the computational time.

\section{3-D NUMERICAL SIMULATION OF COMPRESSOR}

Hub and shroud profile modification is the final target of this process. The quasi-3D analysis code, which is incorporated into the inverse design algorithm, is based on inviscid flow and cannot estimate the performance. A fully $3 \mathrm{D}$ viscous code is used to analyze the flow field of the compressor for evaluation of the quasi-3D analysis results. Also, it can be used for performance prediction of the current and modified compressor.

\subsection{Numerical Method and Boundary Conditions}

The centrifugal compressor is composed of a radial impeller with 25 blades and a radial vaned diffuser with 24 blades. The first and most important step of the numerical simulation of flow inside the compressor is the geometry definition and grid generation. This step is usually the most timeconsuming part. Selection of grid type and locations for grid refinements are the next important tasks. In this simulation, structural elements are used for grid generation of impeller and diffuser. Finer grids are used for zones having steep gradients such as points adjacent to the walls and blades.

The Reynolds-Averaged Navier-Stokes equations (RANS) which describe the conservation of mass, momentum and energy, are solved by means of a finite volume method. Discretization of the equations is done via a coupled implicit method in which the energy, momentum and mass equations are solved simultaneously. The Reynolds stress terms in the momentum transport equations are resolved using the shear-stress transport (SST) turbulence model. This turbulence model is developed to blend the robust and accurate formulation of the $\mathrm{k}-\omega$ model in the nearwall region with the free-stream independence of the $\mathrm{k}-\varepsilon$ model in the far field (Menter et al. 2003).

Using the mixing plane interface model, computational domain is divided into stationary and moving zones. This utilizes relative motion between the various zones to exchange calculated values between zones. To complete the model in rotating zones, the coriolis and centrifugal accelerations are added to the momentum equations. The mixing plane method is a "non-physical snapshot approach" which cannot be compared to a snapshot of a transient simulation. This is because the solution does not know anything about what happened before, but it mixes the impeller flow state into the diffuser. The mixing plane model on the other hand has the advantage that only one pitch of the impeller and diffuser has to be modeled. Figure 12 shows one pitch of the impeller and diffuser with their grids.

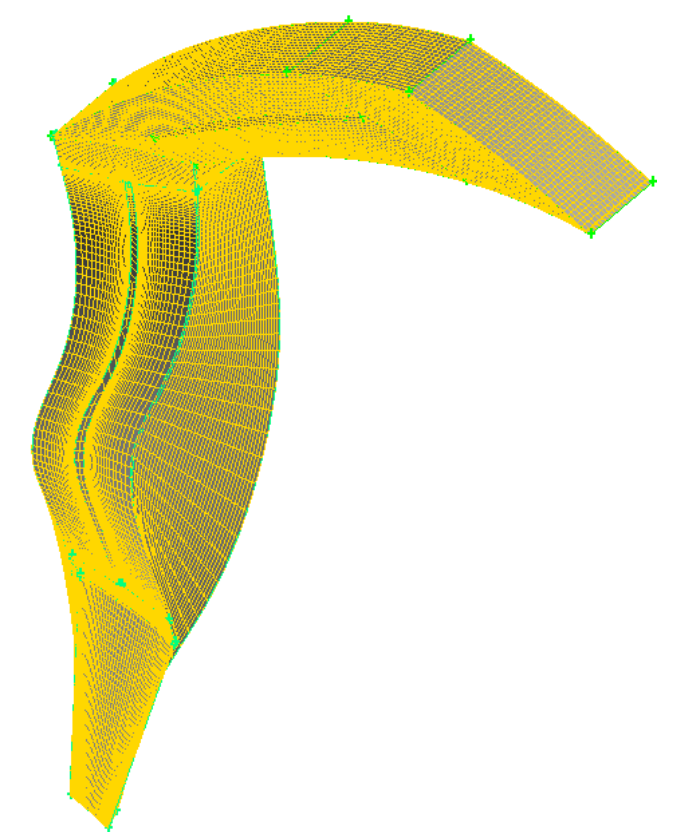

Fig. 12. Compressor Geometry with its grid

The boundary conditions for this simulation include mass flow rate and total temperature at the inlet, average static pressure at the outlet, no slip condition for stationary walls, zero relative velocity with respect to the rotating zone for rotating walls, mixing plane for interface between the impeller, diffuser and periodic boundary condition. It is noticeable that the quantities of the boundary conditions are set from experimental measurements at the inlet and outlet of the compressor while the compressor works as a part of the gas turbine along its operation line.

Theoretically, the errors in the solution related to the grid must disappear by increasing mesh resolution (Ferziger et al. 1996). The compressor pressure ratio and efficiency at nominal flow conditions are taken as the parameters to evaluate three grid configurations (Fig. 13), and to determine the influence of mesh size on the solution. The selected convergence criterion was the attainment to a constant value for drag, lift and moment coefficients of walls. In Fig. 13, it is observed how the calculated compressor pressure ratio and efficiency reach an asymptotic value as the number of elements increases. According to this figure, the grid B (648538 elements) was considered to be sufficiently fine to ensure mesh independency. The $y+$ value 
changes from 30 to 130 for grid $\mathrm{B}$.
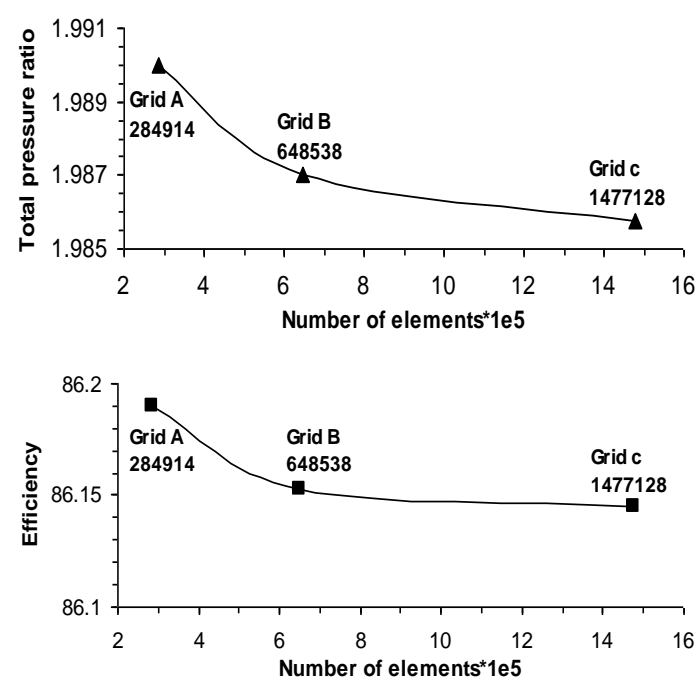

Fig. 13. Effect of grid size on pressure ratio and efficiency of the compressor

\subsection{Current Compressor Flow Field}

The 3D numerical analysis is accomplished in different rotational speeds. In this section, the flow field contours are shown in maximum efficiency.

Figures 14, 15 and 16 respectively show the static

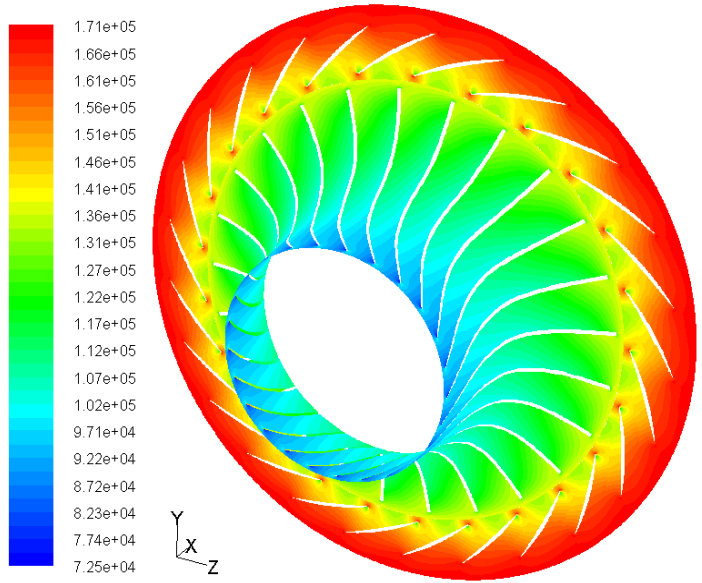

Fig. 14. Static pressure contour on the spanwise section pressure, absolute Mach number and stagnation pressure field at half spanwise section between the hub and shroud in design conditions. As can be seen in Fig. 14, the static pressure rises from the impeller inlet toward the diffuser outlet. This is due to increase in section area inside the impeller and diffuser passage and, increase in centrifugal force through the impeller. Figure 15 shows that Mach number at the impeller outlet and diffuser inlet is near 1 and thus, any increase in rotational speed can choke the compressor.

In Fig. 16, it is clear that the stagnation pressure increases through the impeller because of work done by the impeller on the fluid. In the diffuser, a slight decrease in stagnation pressure is observed because of friction loss. Moreover, the stagnation pressure decreases behind the diffuser blades due to their wake region.

Figures 17, 18 and 19 show the contours of static pressure, reduced pressure and relative velocity field on the periodic section of impeller in design conditions. These three figures can be compared with Figs. 8, 9 and 10. In Fig. 19, decrease in relative velocity near the shroud is related to the growth of boundary layer thickness due to steep adverse gradient of reduced pressure. It shows that the pressure distribution along the shroud needs to be modified to improve the flow loss.

Other contours of current compressor in comparison with the modified compressor contours will be presented in section 6 .
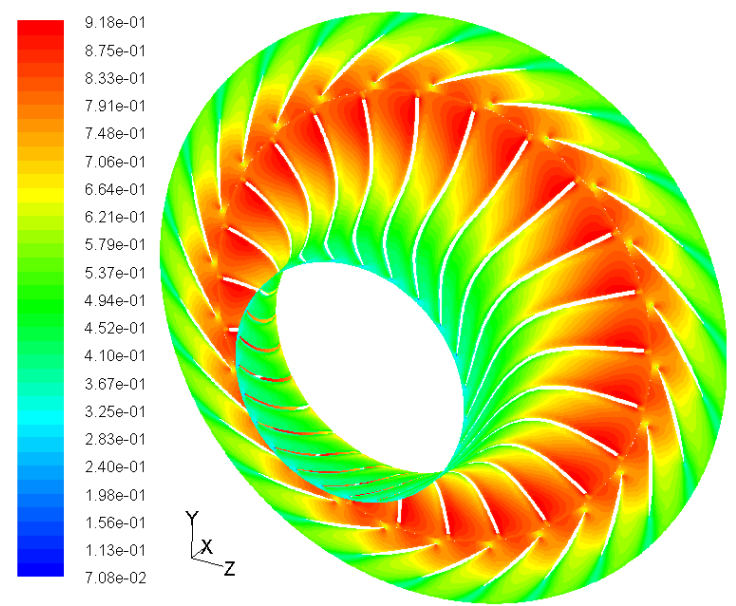

Fig. 15. Mach number contour on the spanwise section 

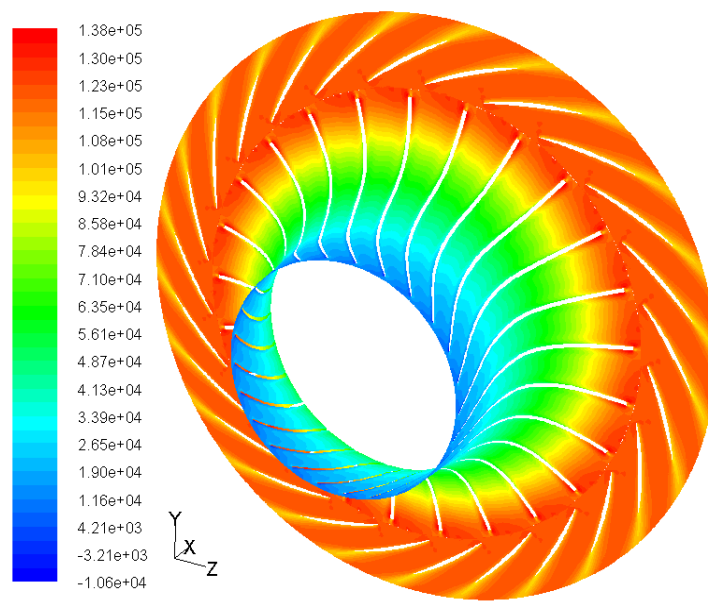

Fig. 16. Stagnation pressure contour on the spanwise section
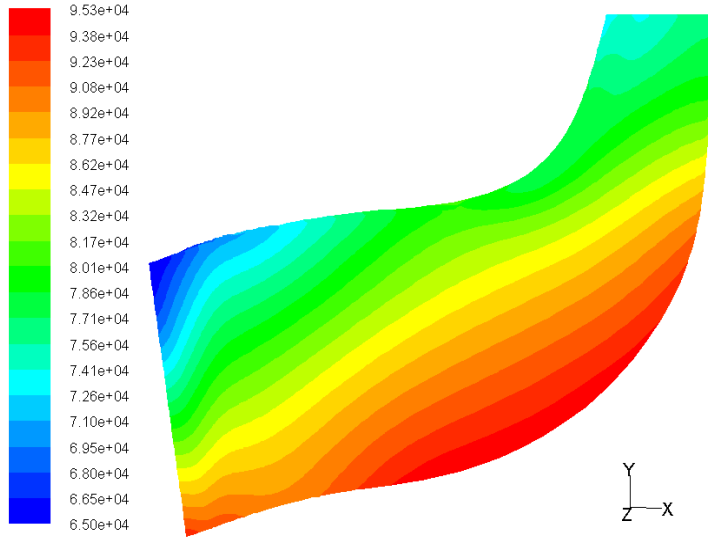

Fig. 18. Reduced pressure contour on the periodic section

\subsection{Validation of Quasi-3D Analysis with 3D Numerical Simulation}

Before combining the quasi-3D code with BSA design algorithm, it is necessary to validate the quasi3D analysis results with 3D numerical simulation.

In Fig. 20, the hub pressure distribution resulted from quasi-3D analysis is compared with results of 3D numerical simulation. Although viscous and 3D effects and clearance are ignored in quasi-3D analysis, it well agrees with 3D simulation. In Fig. 21, the pressure distribution on the shroud calculated by quasi-3D, 3D numerical simulation and experimental measurements are compared. Except next to the inlet and outlet, a good agreement is observed through the passage. The deviation in the inlet area is due to flow deflection at the blade leading edge. The 3D numerical simulation shows that a momentum decrease due to flow losses exists at the end of the shroud wall. Because the losses due to viscous and 3D flow field inside the impeller are not considered in the quasi-3D analysis, this deviation at the end of the shroud can be justified. Also, in order to validate the

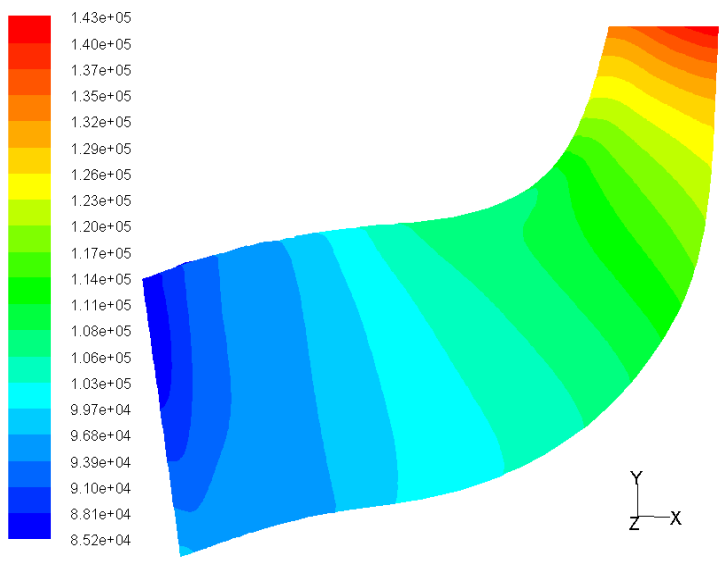

Fig. 17. Static pressure contour on the periodic section

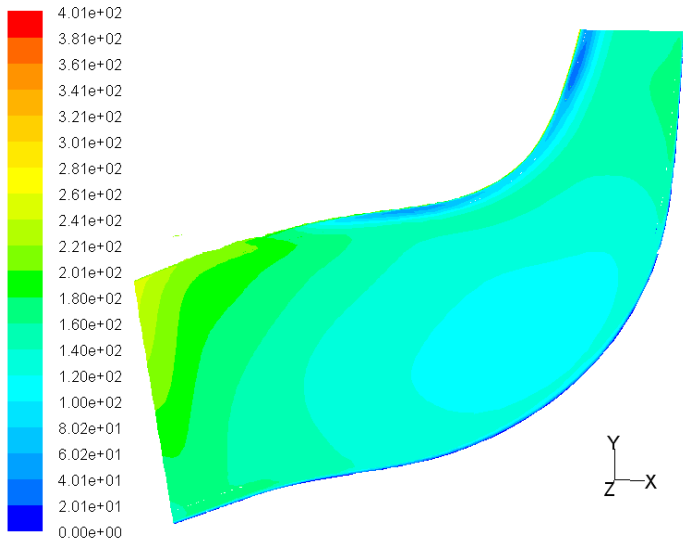

Fig. 19. Relative velocity contour on the periodic section

numerical results, the static pressure is measured at four points on the shroud at Sharif Gas Turbine Lab. The numerical results show good agreement with experimental measurements.

In Fig. 22, a comparison between the quasi-3D and 3D analysis results is accomplished for the reduced pressure on the hub and shroud. Similar to the previous figures, good agreement exists except beside the inlet and outlet. It shows that the modification of meridional plane by combining BSA and quasi-3D code will improve the impeller performance although the viscous and $3 \mathrm{D}$ effects are ignored in the design process.

\section{COMPRESSOR EXPERIMENTAL SETUP}

In this research, the centrifugal compressor is tested as a component in a gas turbine. The necessary instruments to measure mass flow and pressures in different parts of the compressor are added to the gas turbine test setup. The schematic of experimental 
arrangement is shown in Fig. 23.

The measurements are done at four areas namely; impeller inlet, shroud wall, impeller outlet and diffuser outlet. The rotational speed is measured by a magnetic pick-up and mass flow rate by pressure difference across the belmouth at the gas turbine inlet. A total of 11 static pressure taps, 6 stagnation pressure probes and 8 thermocouples are used for measuring thermodynamic parameters at different

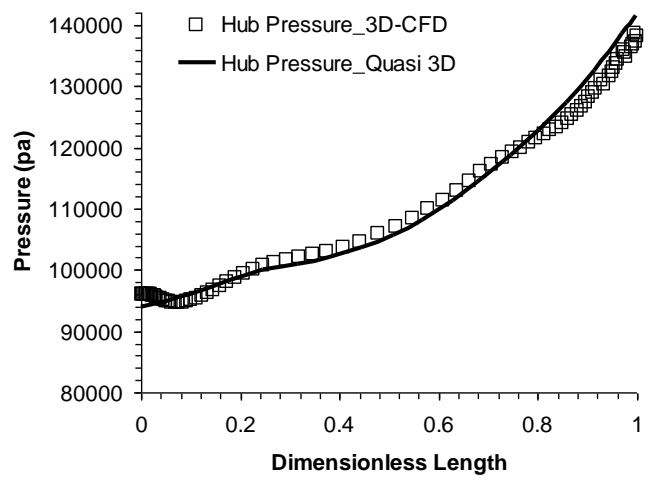

Fig. 20. Pressure distribution on the hub due to the quasi 3D and $3 \mathrm{D}$ analysis

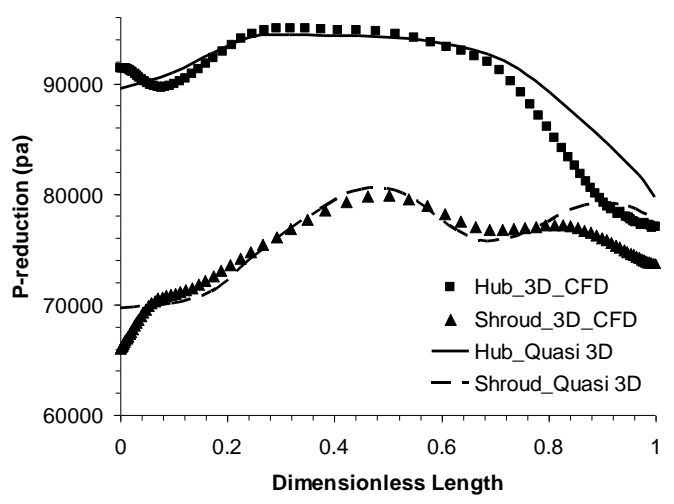

Fig. 22. Reduced pressure distribution on the hub and shroud due to the quasi 3D and 3D analysis

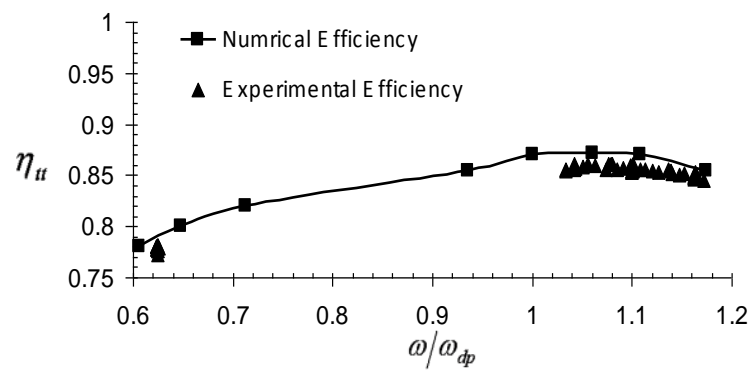

Fig. 24. Numerical and experimental efficiency positions. The variation of total to total isentropic efficiency and total pressure ratio versus the normalized rotational speed are extracted from the experiments.

The experimental performance results are used for validation of 3D numerical simulation results. In Figs. 24 and 25 the numerical performance is compared with the experimental performance and shows good agreement.

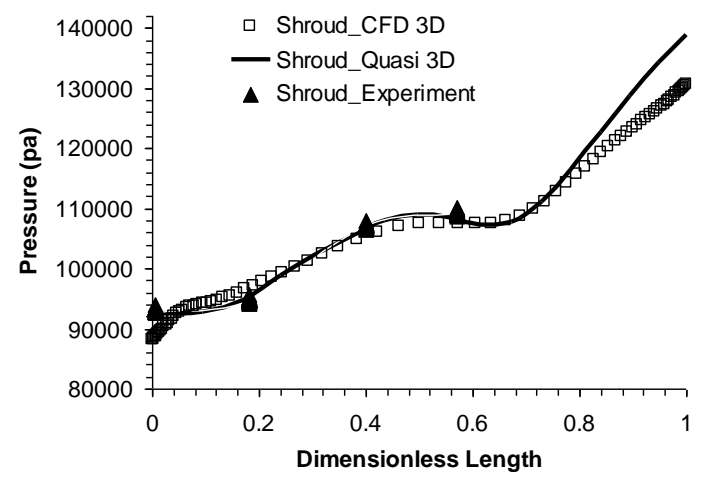

Fig. 21. Pressure distribution on the shroud due to the quasi $3 \mathrm{D}, 3 \mathrm{D}$ analysis and experiment

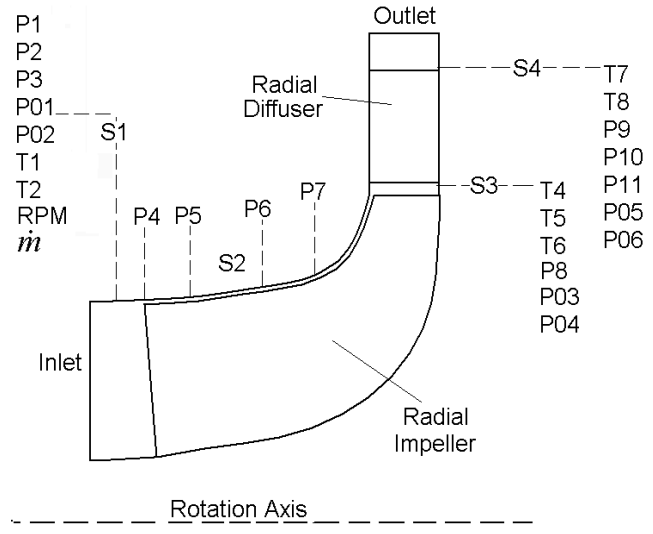

Fig. 23. Schematic of experimental setup for the centrifugal compressor of the gas turbine

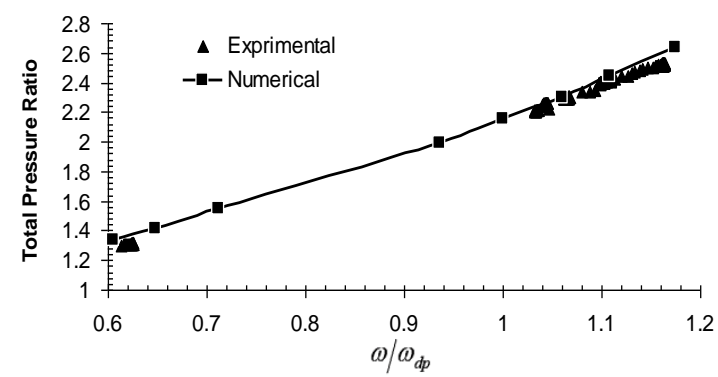

Fig. 25. Numerical and experimental total pressure ratio 


\section{DESIGN EXAMPLES}

After validating the quasi-3D analysis results, the BSA design algorithm is incorporated into the quasi3D code and the hub-shroud profile of the impeller is obtained by modifying the reduced pressure distribution along the hub and shroud.

In Fig. 26, having modified the $\mathrm{CP}_{\mathrm{r}} \mathrm{D}$, the corresponding shape of the hub and shroud is obtained. In this design (design $\mathrm{A}$ ), the additional adverse pressure gradient along the shroud is removed but, the inlet and outlet pressures are not changed. In other words, keeping the area ratio fixed, it is tried to make it smooth. Moreover, as seen in Fig. 26 , the axial length of the modified impeller is reduced by $5 \%$.

In design $\mathrm{B}$ shown in Fig. 27, a slight increase in outlet pressure is seen that causes the area ratio to be increased. Also, the adverse pressure gradient along the shroud is lower than that of design A. This will reduce the boundary layer thickening on the shroud. It is clear that the deflection angle from the inlet to the outlet depends on the surrounded area between the reduced pressure distributions along the hub and shroud. As seen in Fig. 27, this surrounded area for the modified pressure has been increased compared to the original case.

In design C (Fig. 28), it is tried to reach to a minimum adverse pressure gradient on the shroud in such a way that the inlet and outlet pressure and, the surrounded area between the hub and shroud pressure remain unchanged, hence the deflection is unchanged. In this case, the axial length of the modified impeller is reduced by $10 \%$. The modifications in design $\mathrm{C}$ will decrease the boundary layer losses, and also the blade effective area (meridional plane area) and average pressure level of the blade. Hence, it causes the blade work to be transferred to the fluid in a lower pressure level and on a smaller area. Therefore, it is expected that the pressure ratio of the impeller in design $\mathrm{C}$ decreases.

In cases A, B and C, the impeller hub and shroud were modified resulting a change in the axial length of the impeller. It is clear the separation along the shroud is more probable than along the hub, therefore, to improve the meridional plane geometry with a minimum change in geometry and without change in the axial length, the hub geometry is kept fixed and the shroud is to be modified. As a result, the reduced pressure distribution along the hub is out of control and is obtained automatically.

To specify the $\mathrm{TP}_{\mathrm{r}} \mathrm{D}$ along the shroud, two important points should be considered. First, the pressure applied to the fluid in rotating zone is the reduced pressure. Just as the fluid exits from a rotating zone, the pressure applied to the fluid changes from the reduced pressure to the static pressure. In other words, the fluid is confronted with a pressure jump of $1 / 2 \rho r^{2} \omega^{2}$ that causes the wake region at the impeller outlet to be intensified. To overcome this pressure jump, it is necessary that the fluid be accelerated before exiting the impeller. The slope of the reduced pressure distribution on the hub is negative and the fluid is accelerated before exiting the impeller. The main problem is related to the shroud along which the reduced pressure increases. The current distribution of the reduced pressure along the shroud has an advantage that the reduced pressure slope at the impeller outlet is negative and the fluid is accelerated before exiting. As seen in the $\mathrm{TP}_{\mathrm{r}} \mathrm{D}$ of the design $\mathrm{D}$ (Fig. 29), the reduced pressure along the shroud increases and suddenly decreases near the end part.

The second point is that in most efficient diffusers, the passage experiences a high pressure loading at the first part followed by a moderate one toward its end. As seen in the $\mathrm{TP}_{\mathrm{r}} \mathrm{D}$ of design $\mathrm{D}$ (Fig. 29), this physical base is followed.
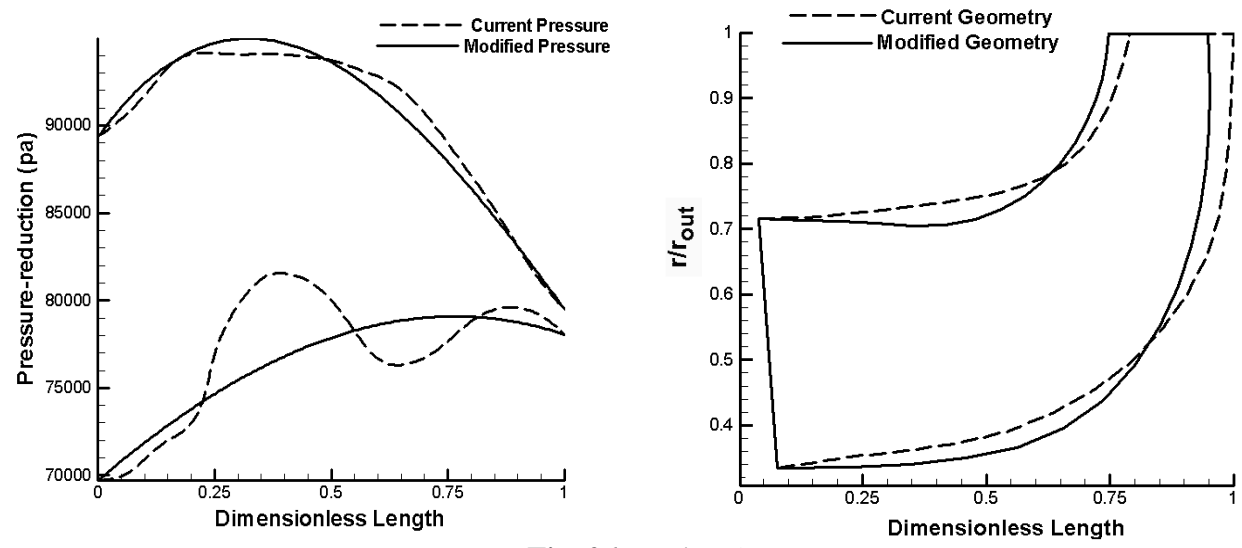

Fig. 26. Design A 

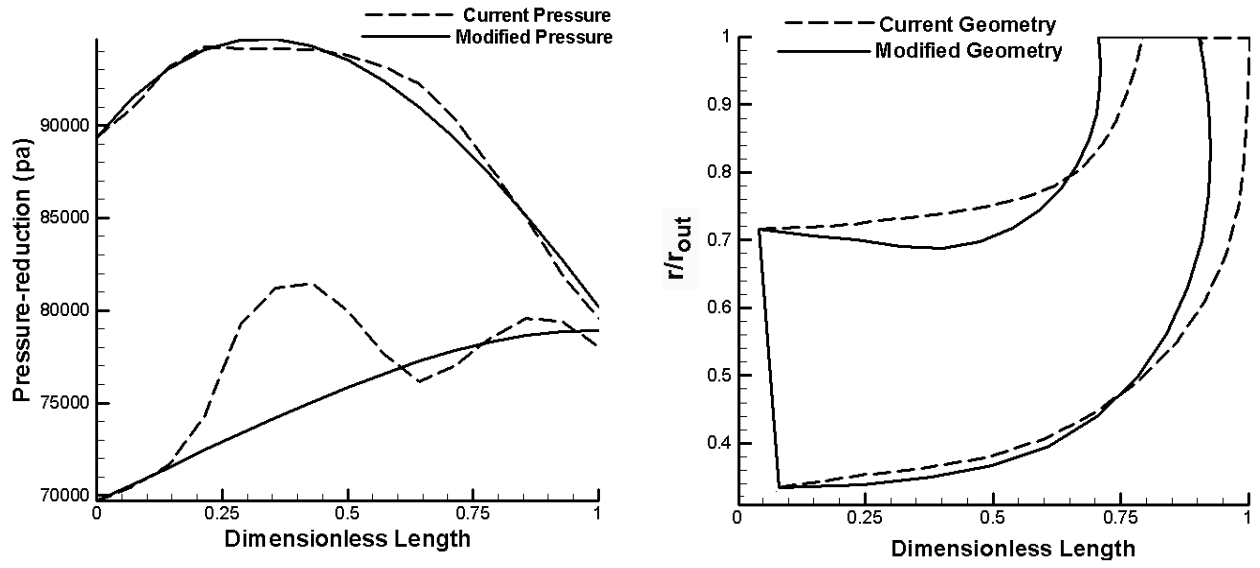

Fig. 27. Design B
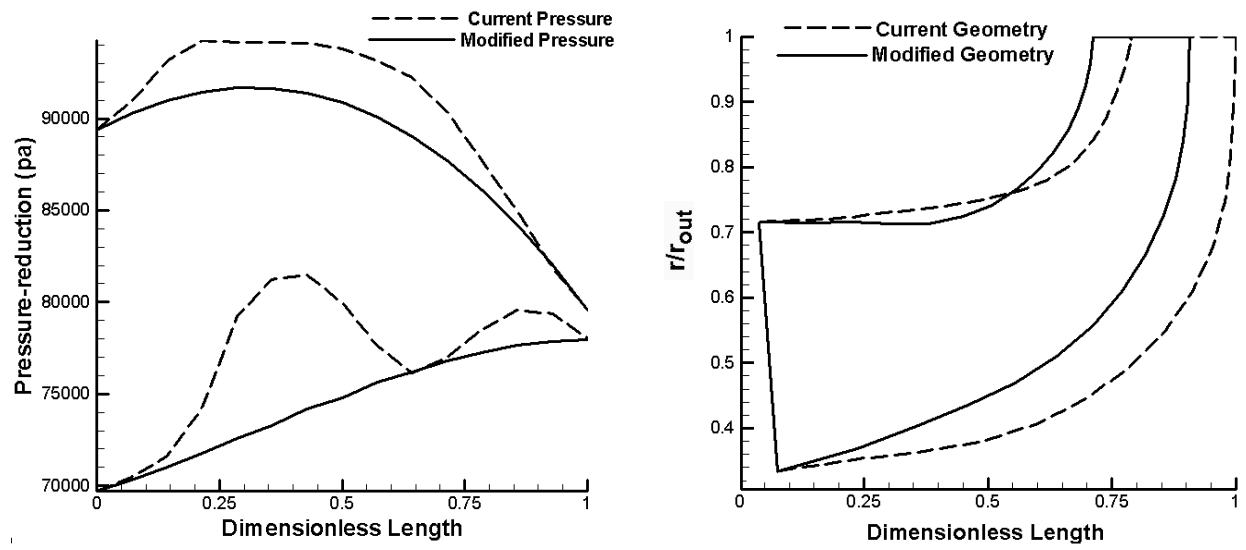

Fig. 28. Design C
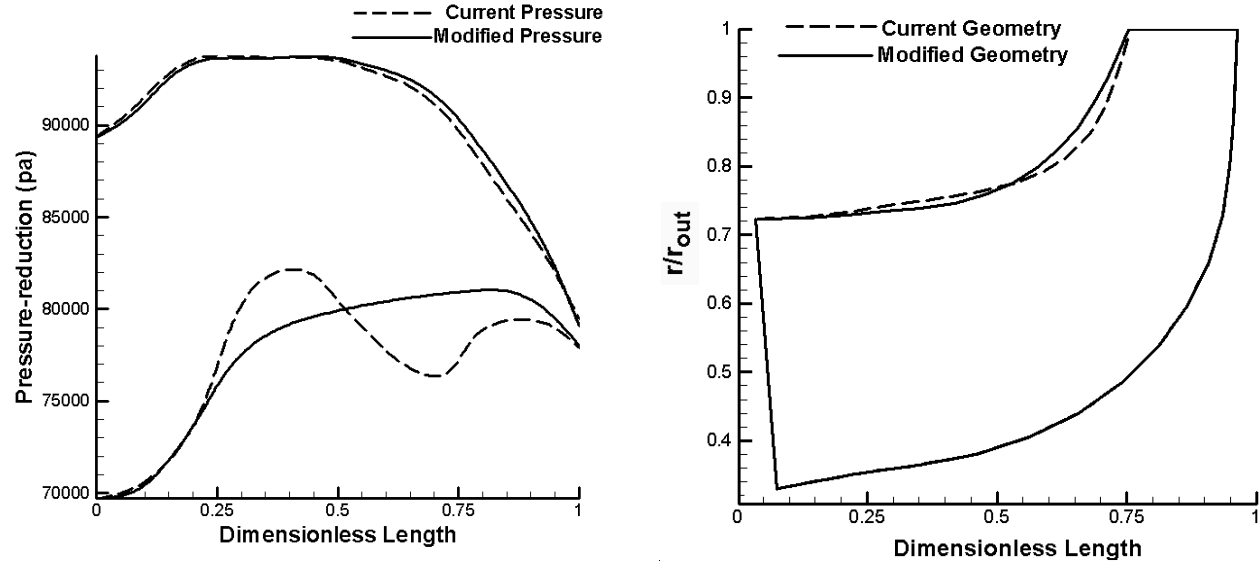

Fig. 29. Design D

The 3D numerical simulation of design $\mathrm{D}$ shows that the efficiency is improved by 0.6 percent compared to the original design. The increment of the efficiency versus the normalized rotational speeds is shown in Fig. 30. It is noticeable that, there is no difference between the grid generation of the modified impeller and that of the current impeller in the $3 \mathrm{D}$ numerical simulation. In Figs. 31-35, the flow field contours of the modified geometry (design D) are compared with that of the current geometry. Figure 31 shows that the relative velocity decrement at the end part of the shroud is improved for design D. Figure 32 shows the relative stagnation pressure contour on the periodic surface of the impeller, indicating the energy losses. 
As seen, the relative stagnation pressure loss near the shroud is improved. In Fig. 33, the relative stagnation pressure contour is shown at the impeller outlet for the current impeller and design D. It shows that the energy losses near the shroud have been decreased. The relative velocity contour at the impeller outlet is shown in Fig. 34. The wake region in the shroud area is weakened for design. Also, the wake behind the blade is clearly seen in this figure. The radial velocity contour at the impeller outlet is shown in Fig. 35, indicating the removal of small reverse flow region with negative radial velocity in design $\mathrm{D}$ compared to the original design.
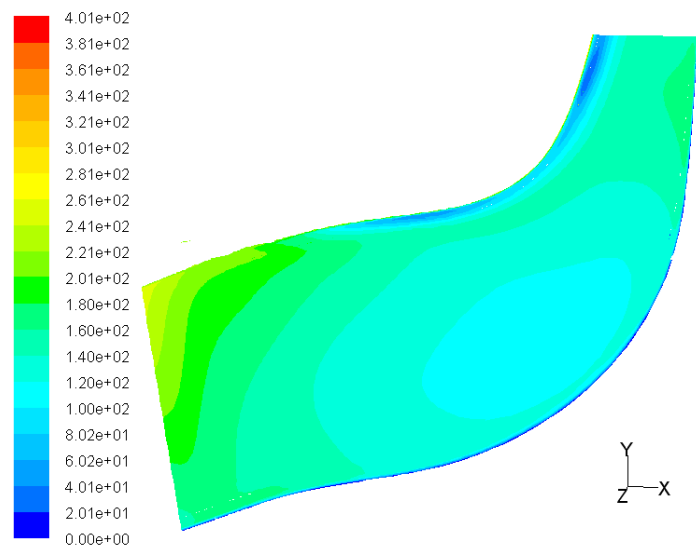

a) Current Impeller

Fig. 31. Relative velocity contour on the periodic surface of the impeller
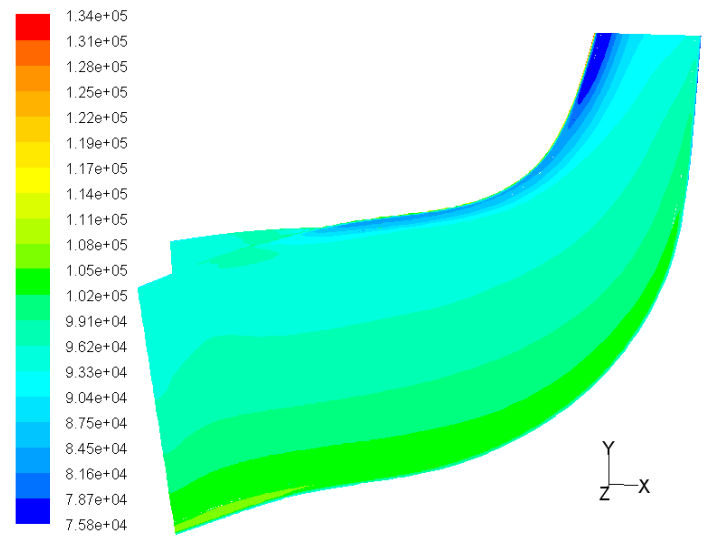

a) Current Impeller

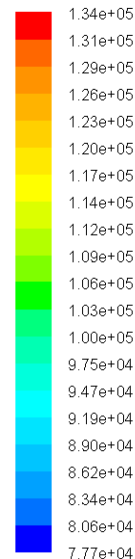

Fig. 32. Relative stagnation pressure contour on the periodic surface of the impeller

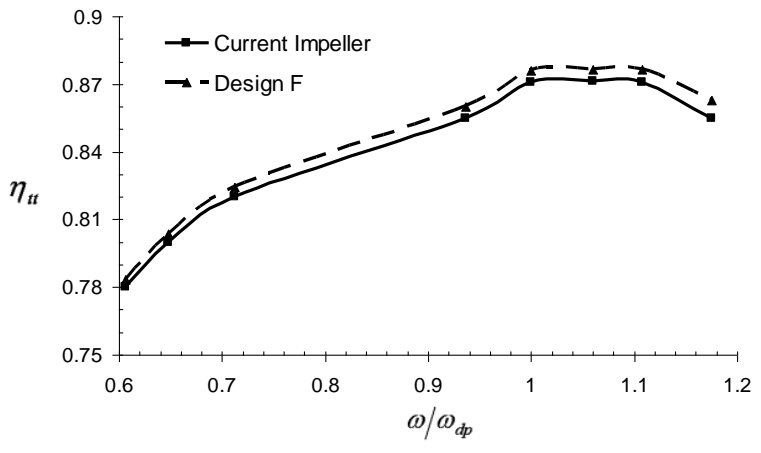

Fig. 30. Efficiency improvement versus the rotational speed
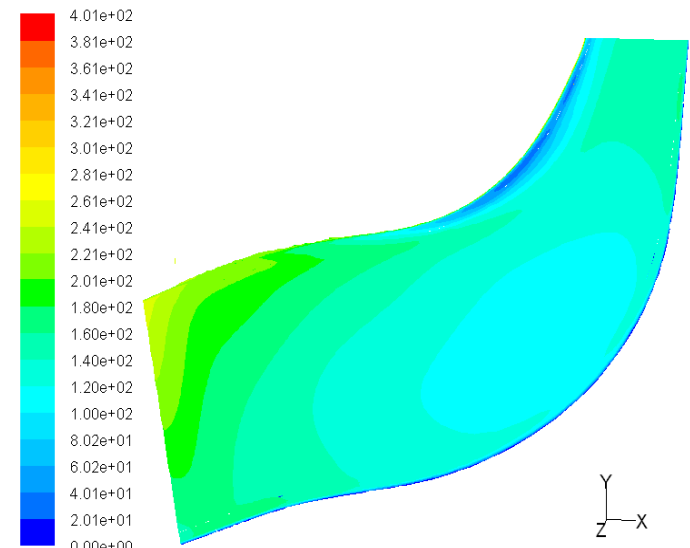

b) Design $F$ 


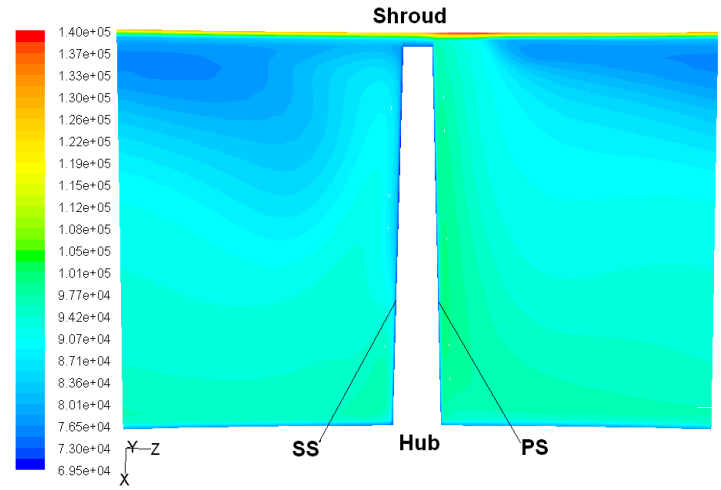

a) Current Impeller

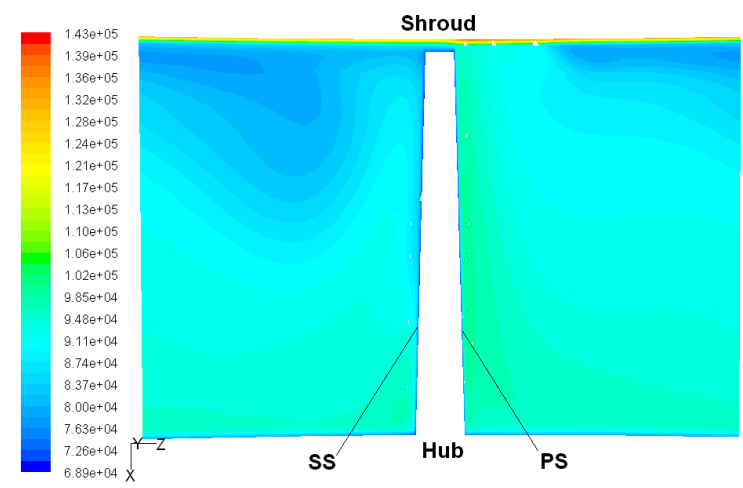

b) Design $F$

Fig. 33. Relative stagnation pressure contour on the impeller outlet

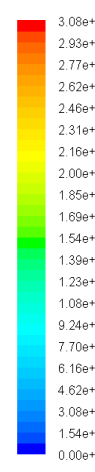

$3.086+02$
$2.93 e+02$
$2.77 e+02$
$2.62 e+02$
$2.46 e+02$
$2.31 e+02$
$2.16 e+02$
$2.00 e+02$
$1.85 e+02$
$1.69 e+02$
$1.54 e+02$
$1.39 e+02$
$1.23 e+02$
$1.08 e+02$
$9.24 e+01$
$7.70 e+01$
$6.16 e+01$
$4.62 e+01$
$3.08 e+01$
$1.54 e+01$
$0.00 e+00$

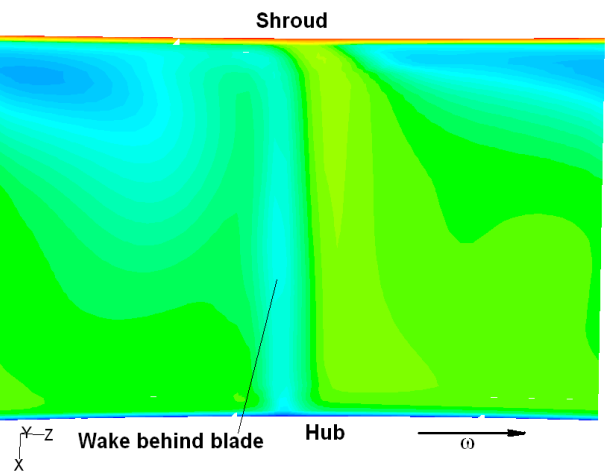

a) Current Impeller

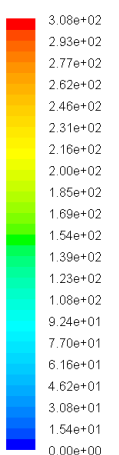

$000+00$

Fig. 34. Relative velocity contour on the impeller outlet

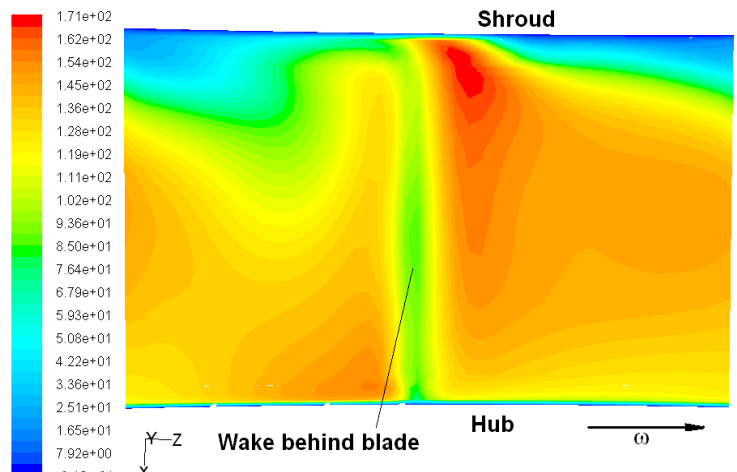

a) Current Impeller

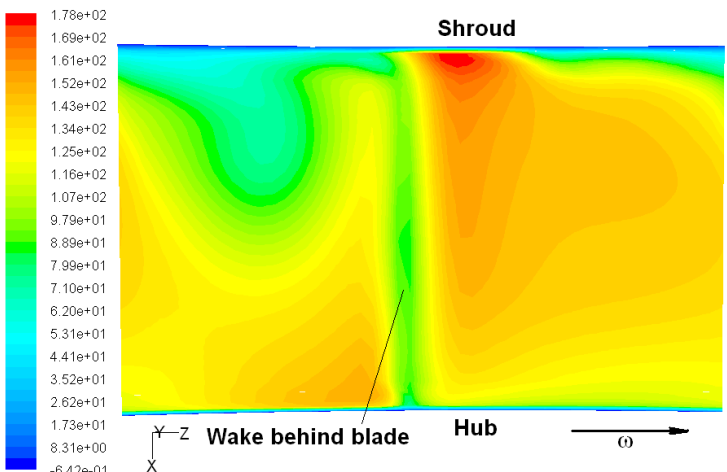

b) Design F

Fig. 35. Radial velocity contour on the impeller outlet

\section{CONCLUSIONS}

The BSA turns the inverse design problem into a fluid-solid interaction scheme that is a physical base analysis. The BSA is a quick converging method and can efficiently utilize commercial flow analysis software as a black-box. In this research, the BSA design procedure was combined with a quasi-3D analysis code for designing the hub and shroud profiles of a centrifugal compressor impeller. For the convergence of the BSA in a rotating zone, the difference between the CPrD and TPrD should be applied to the flexible wall at each shape modification step. Considering a TPrD on the shroud featuring a passage high loading at the first part followed by a moderate one at the middle part and terminated to a negative one at the end, the design procedure converges to a shroud profile that improved the compressor efficiency by 0.6 percent. 
M. Nili-Ahmadabadi et al. / JAFM, Vol. 7, No. 2, pp. 329-344, 2014.

\section{REFRENCES}

Ghadak, F. (2005). A Direct Design Method Based on the Laplace and Euler Equations, Ph. D. Thesis, Sharif University of Technology, Tehran, Iran.

Cheng, C. H. and C. Y. Wu (2000). An approach combining body fitted grid generation and conjugate gradient method for shape design in heat conduction problems. Numerical Heat Transfer Part B 37(1), 69-83.

Jameson, A. (1994). Optimal design via boundary control. Optimal Design Methods for Aeronautics, AGARD, 3.1 - 3.33.

Kim, J. S. and W. G. Park (2000). Optimized inverse design method for pump impeller. Mechanics Research Communications 27(4), 465-473.

Dedoussis, V., P. Chaviaropoulos and K. D. Papailiou (1993). Rotational compressible inverse design method for two-dimensional internal flow configurations. AIAA Journal 31(3), 551-558.

Demeulenaere A. and R. V. D. Braembussche (1998). Three-dimensional inverse method for turbomachinary blading design. ASME Journal of Turbomachinary 120(2), 247-255.

De Vito, L. and R.V.D. Braembuussche (2003). A novel two-dimentional viscous inverse design method for turbomachinery blading. Transactions of the ASME 125, 310-316.

Leonard, O. and R.V.D. Braembuussche (1997). A two-dimensional Navier Stokes inverse solver for compressor and turbine blade design. Proceeding of the IMECH E part A, Journal of Power and Energy 211, 299-307.

Henne, P. A. (1980). An inverse transonic wing design method. AIAA Paper 80-0330.

Volpe, G. (1989). Inverse design of airfoil contours: constraints, numerical method applications. See AGARD, Paper 4.

Barger, R. L. and C. W. Brooks (1974). A streamline curvature method for design of supercritical and subcritical airfoils. NASA TN D-7770.

Garabedian, P. and G. McFadden (1982). Design of supercritical swept wings. AIAA Journal 30(3), 444-446.

Malone, J., J. Vadyak and L. N. Sankar (1986). Inverse aerodynamic design method for aircraft components. Journal of Aircraft 24(1), 8-9.
Malone, J., J. Vadyak and L. N. Sankar (1986). A technique for the inverse aerodynamic design of nacelles and wing configurations. AIAA Paper 85-4096.

Campbell, R. L. and L. A. Smith (1987). A hybrid algorithm for transonic airfoil and wing design. AIAA Paper 87-2552.

Bell, R. A. and R. D. Cedar (1991, October). An inverse method for the aerodynamic design of three-dimensional aircraft engine nacelles. In G.S. Dulikravich (Ed.), Proceedings of the Third International Conference on Inverse Design Concepts and Optimization in Engineering Sciences ICIDES-III, Washington, D.C., USA 405-417.

Malone, J. B., J. C. Narramore and L. N. Sankar (1989). An efficient airfoil design method using the Navier-Stokes equations. AGARD, Paper 5.

Malone, J. B., J. C. Narramore and L. N. Sankar (1991). Airfoil design method using the NavierStokes equations. Journal of Aircraft 28(3), 216224.

Takanashi, S. (1985). Iterative three-dimensional transonic wing design using integral equations. Journal of Aircraft 22, pp. 655-660.

Hirose, N., S. Takanashi, and N. Kawai (1987). Transonic airfoil design procedure utilizing a Navier-Stokes analysis code. AIAA Journal 25(3), 353-359.

Dulikravich, G. S. and D. P. Baker (1999). Aerodynamic shape inverse design using a Fourier series method. AIAA Paper 99-0185.

Aungier, R. H. (2000). Centrifugal Compressor, A Strategy for Aerodynamic Design and Analysis. New York, USA, ASME Press.

Vanco, M. R. (1972). Fortran program for calculating velocities in the meridional plane of a turbomachine. NASA TN D-6701.

Zangeneh, M., W. N. Dawes and W. R. Hawthorne (1988). Three-dimensional flow in radial-inflow turbine. ASME paper No. 88-GT-103.

Nili-Ahmadabadi, M., M. Durali, A. Hajilouy and F. Ghadak (2009). Inverse design of 2D subsonic ducts using flexible string algorithm. Inverse Problems in Science and Engineering 17(8), 1037-1057.

Nili-Ahmadabadi, M., A. Hajilouy, M. Durali, and F. Ghadak (2009). Duct design in subsonic \& supersonic flow regimes with \& without Shock using flexible string algorithm. Proceedings of 
M. Nili-Ahmadabadi et al. / JAFM, Vol. 7, No. 2, pp. 329-344, 2014.

ASME Turbo Expo 2009, Florida, USA, GT2009-59744.

Nili-Ahmadabadi, M., A. Hajilouy, M. Durali, and F. Ghadak (2008, August). Inverse design of 2D ducts for incompressible viscous flow using flexible string algorithm. Proceedings of the 12th Asian Congress of Fluid Mechanics, Daejeon, Korea.

Menter, F. R., M. Kuntz and R. Langtry (2003). Ten years of industrial experience with the SST turbulence model. In K. Hanjalic, Y. Nagano and M. Tummers, (Ed.), Turbulence, Heat and Mass Transfer 4, 625-632.

Ferziger, J. H. and M. Peric (1996). Computational Methods for Fluid Dynamics. Berlin, Germany, Springer. 